\section{Urabstimmung TARMED}

Die Delegierten der Ärztekammer haben an ihrer ausserordentlichen Sitzung vom 13. Dezember 2001 die Durchführung einer Urabstimmung mit 133 Stimmen - ohne Gegenstimme und bei drei Enthaltungen - beschlossen, und damit das erforderliche Quorum erreicht, mit welchem die Urabstimmung gemäss Art. 24, Abs. 3 der Statuten der Verbindung der Schweizer Ärztinnen und Ärzten unverzüglich an die Hand genommen werden kann.

Den Mitgliedern werden folgende Fragen zur Abstimmung gebracht:

A Wollen Sie die Tarifstruktur TARMED Version 1.1 vorbehältlich der Umsetzung der Vereinbarung zum Reengineering, namentlich dem REII-Konzept, bis zum 30. Juni 2003 und vorbehältlich einer einvernehmlich vereinbarten Regelung des Datentransfers zwischen Ärztinnen/Ärzten und Versicherern als Einführungstarifstruktur für die kantonalen KVG-Tarife annehmen?

B Wollen Sie dem KVG-Rahmenvertrag TARMED vom 21. November 2001 vorbehältlich einer einvernehmlich vereinbarten Regelung des Datentransfers zwischen Ärztinnen/Ärzten und Versicherern zustimmen?

C Wollen Sie dem UV-/MV-/IV-Tarifvertrag, beinhaltend einen Starttaxpunktwert von Fr. 1.- zustimmen?

Hinsichtlich der Tarifstruktur (Frage A) stimmte die Ärztekammer mit 124 Stimmen - bei 29 Gegenstimmen und 12 Enthaltungen - dem Antrag des Zentralvorstandes zu, die Version 1.1 als Einführungsversion für die Arzttarife im KVG-Bereich anzunehmen, sofern die Vereinbarung zum Reengineering, namentlich das RE II-Konzept, bis zum 30. Juni 2003 umgesetzt werde.
Die Zustimmung zum UV-/MV-/IV-Tarifvertrag (Frage C) hatten die Delegierten bereits an der Ärztekammer vom 4./5. Mai 2001 beschlossen, ergänzt um die Bedingung, dass die gleichen Konditionen auch für den ambulanten Spitalbereich gelten müssen.

Dem Antrag des Zentralvorstandes zur Zustimmung zum Rahmenvertrag KVG (Frage B), vorbehältlich einer einvernehmlich vereinbarten Regelung des Datentransfers zwischen Ärztinnen/Ärzten und Versicherern, stimmte die Ärztekammer mit 69 Stimmen - bei 9 Gegenstimmen und 7 Enthaltungen - zu. Da das statutarisch geforderte Quorum bei dieser Abstimmung nicht mehr erreicht werden konnte, gilt der Entscheid indessen als konsultativ.

Derzeit wird mit Hochdruck an der Vorbereitung der Urabstimmung gearbeitet, denn den Mitgliedern der FMH sollen Anfang Februar 2002 die Abstimmungsunterlagen zugestellt werden. Diese enthalten nebst dem Stimmzettel, den statutarisch geforderten Erläuterungen sowie der Würdigung durch den Zentralvorstand eine CD-ROM mit den Abstimmungsmaterialien. Sämtliche Unterlagen werden im Vorgang zur Abstimmung in der Schweizerischen Ärztezeitung publiziert. Wir bitten die stimmberechtigten Mitglieder, den entsprechenden Ausgaben Nr. 4 und 5 sowie den jeweils beigelegten Supplementa Aufmerksamkeit zu schenken.

Abstimmungsdatum ist der 4. März 2002, anschliessend werden die Stimmen ausgezählt und das Resultat veröffentlicht.

Wir bitten alle FMH-Mitglieder, von ihrem Stimmrecht Gebrauch zu machen. Namens des Zentralvorstandes der FMH empfehlen wir, die Einführung von TARMED als Vertragstarif für ambulante Leistungen im Sozialversicherungsbereich nun ein für allemal zu beschliessen.

Dr. Hans Heinrich Brunner, Präsident FMH

Annamaria Müller Imboden, Generalsekretärin FMH 